\title{
On the Estimation Problems for Exponentiated Exponential Distribution under Generalized Progressive Hybrid Censoring
}

\author{
Aakriti Pandey \\ Arun Kaushik \\ Sanjay K. Singh \\ Umesh Singh \\ Department of Statistics, Institute of Science, \\ Banaras Hindu University, Varanasi, India-221005

\begin{abstract}
In this article, we considered the statistical inference for the unknown parameters of exponentiated exponential distribution based on a generalized progressive hybrid censored sample under classical paradigm. We have obtained maximum likelihood estimators of the unknown parameters and confidence intervals utilizing asymptotic theory. Entropy measures, such as Shannon entropy and Awad sub-entropy, have been obtained to measure loss of information owing to censoring. Further, the expected total time of the test and expected number of failures, which are useful during the execution of an experiment, also have been computed. The performance of the estimators have been discussed based on mean squared errors. Moreover, the effect of choice of parameters, termination time $T$, and $m$ on the ETTT and ETNFs also have been observed. For illustrating the proposed methodology, a real data set is considered.
\end{abstract}

Keywords: entropy, maximum likelihood estimates, generalized progressive hybrid censoring, total expected number of failures, total time of the test.

\section{Introduction}

To capture the true behaviour of a system, a number of probability models are available in statistical literature. Among these, exponential distribution is the oldest, simplest, and widely used model for lifetime data. Exponentiated exponential distribution(EED) is a generalization of exponential distribution, introduced by Gupta and Kundu 1999. Later, Gupta and Kundu (2001a) showed that EED is a good alternative to gamma and Weibull distributions, and have further discussed its advantages over these two commonly used distributions. The estimation of the parameters of EED have been attempted by Gupta and Kundu (2001b). EED is endowed with several desirable properties, including good interpretations, and can be easily generated. All these properties point to why it is widely used. Taking cognisance of the facts discussed above, EED is considered in the present article. The probability density function 
of exponentiated exponential distribution is given as,

$$
f(x \mid \alpha, \beta)=\alpha \beta e^{-\beta x}\left(1-e^{-\beta x}\right)^{\alpha-1} ; x \geq 0, \alpha, \beta>0
$$

The cumulative distribution function and survival function are given by

$$
F(x \mid \alpha, \beta)=\left(1-e^{-\beta x}\right)^{\alpha}
$$

and

$$
S(x \mid \alpha, \beta)=1-\left(1-e^{-\beta x}\right)^{\alpha} ; x \geq 0, \alpha, \beta>0,
$$

respectively. The corresponding hazard function is given by

$$
h(x \mid \alpha, \beta)=\frac{\alpha \beta e^{-\beta x}\left(1-e^{-\beta x}\right)^{\alpha-1}}{1-\left(1-e^{-\beta x}\right)^{\alpha}},
$$

where $\alpha$ is the shape parameter and $\beta$ is the scale parameter of the considered distribution. Due to the scale and shape parameters, it offers some flexibility for analyzing different types of lifetime data. It has an increasing and decreasing hazard rate, depending on the choice of the shape parameter.

In life testing experiments, censoring is a common feature and may occur naturally or owing to some constraint. Among the conventional Type-I, Type-II, and hybrid censoring schemes, the latter is quite popular among life testing experiments, which was introduced by Epstein (1954). The details regarding statistical inferences and applications of exponential distribution under hybrid censoring can be found in Balakrishnan (1996) and Childs et al. (2003). In conventional censoring schemes, the surviving units can be removed only at the terminal point of the experiment. However, there are certain situations where the removal of live units is not confined to the terminal points of the experiment but allowance of intermediate removals is equally important. Kundu and Joarder (2006) and Childs et al. (2008) have considered the Type-I progressive hybrid censoring scheme(Type-I PHCS) in the context of life testing experiments where $n$ identical units are tested with the progressive censoring scheme $\left(R_{1}, R_{2}, \cdots, R_{m}\right)$, where $R_{i}$ is the number of removals at ith failure and the experiment is terminated at time $T^{*}=\min \left\{X_{m: m: n}, T\right\}$, where $T \in(0, \infty)$ and $1 \leq m \leq n$ are fixed in advance and $X_{1: m: n} \leq X_{2: m: n} \leq \cdots \leq X_{m: m: n}$ are ordered failure times from the experiment. One can navigate through Balakrishnan and Aggarwala (2000) and Balakrishnan (2007) to gather information regarding the detailed description about the above-mentioned censoring scheme. The advantage of this censoring scheme is that the test can never go beyond $T$. In this way, this scheme controls the time and cost of the experiment. However, if an unknown average lifetime is comparatively high when compared to stopping time $T$, there is the possibility of lesser than $m$ failures, which eventually reduces the efficiency of the inferences based on such censored data. Keeping this point in mind, Childs et al. (2008) proposed the Type-II progressive hybrid censoring scheme that terminates the experiment at a time $T^{*}=\max \left(X_{m: m: n}, T\right)$ which ensures at least $m$ number of failures and establishes more than a desired level of efficiency. When $X_{m: m: n}>T$, the experiment is terminated at $m^{\text {th }}$ failure with withdrawals occurring at each failure as per the pre-specified progressive scheme $\left(R_{1}, R_{2}, \cdots, R_{m}\right)$. It is important to consider that this may lead to a significant increase in the time of termination. On the other hand, when $X_{m: m: n}<T$, we observe failures upto time $T$. If $D$ represents the number of failures that occur before time $T$, then the progressive censoring scheme, in this case, is $\left(R_{1}, R_{2}, \cdots, R_{m}, R_{m+1}, \cdots, R_{D}\right)$, where $R_{m}=R_{m+1}=\cdots=R_{D}=0$. Thus, we see that in this censoring scheme, the termination time is unknown to the experimenter. From the above discussion, we note that the Type-I hybrid censoring scheme keeps the termination time of the experiment below a prefixed value by forfeiting the efficiency, whereas Type-II hybrid censoring ensures efficiency more than the prefixed level but forfeits the termination time. Therefore, the need for a censoring scheme controlling termination time and efficiency was felt simultaneously. For example, it should ensure the bare minimum number of failures 
and place restrictions on the time as well. Cho et al. (2015b) introduced the generalized progressive hybrid (GPH) censoring scheme, which assimilates the features of both (Type-I PHC and Type-II PHC), as well as irons out their lacunas. Recently, Cho et al. (2015a) computed the estimated entropy of the Weibull distribution for generalized progressive hybrid censored data. The work presented here investigates estimated Shannon entropy, Awad sub-entropy, expected total time of the test, and the expected total number of failure based on a GPH censored data for exponentiated exponential distribution.

The present article is comprised of eight sections. Section 2 describes the considered censoring scheme in detail. Section 3 contains the procedure for obtaining the maximum likelihood estimate. In Section 4, ETTT and ETNF are computed. Section 5 presents the Shannon entropy and Awad sub-entropy estimation for the considered censoring scheme. Section 6 considers a real dataset for illustrating the procedures discussed in the previous sections. Furthermore, a simulation study is conducted and the results are summarized in Section 7. Finally, a concluding remark is presented in Section 8.

\section{The censoring scheme}

Suppose $n$ units are put on test. Keeping in mind the desired minimum efficiency and cost of the items, we prefix integers $k, m \in\{1,2, \cdots, n\}$ such that $k<m$. The lifetimes of the sample units $X_{1}, X_{2}, \cdots, X_{n}$ are independent and identically distributed random variables from a distribution with cumulative density function (cdf) $F(\cdot)$ and probability density function (pdf) $f(\cdot)$ as given in equations(2) and (1), respectively. The removal scheme is such that $R_{i}$ units are to be randomly removed at $i^{t h}$ failure, satisfying the condition $\sum_{i=1}^{m} R_{i}+m=n$. The test continues until the stopping time $T^{*}=\max \left\{X_{k: m: n}, \min \left\{X_{m: m: n}, T\right\}\right\}$ is reached, and, at this stage, the remaining units are removed from the experiment. It may be noted that instead of observing $\mathrm{m}$ number of failures, this scheme guarantees a bare minimum number of $\mathrm{k}$ failures. Let $D$ denotes the number of failures up to time $T$. Thus, three cases arise under this scheme, giving the set of observations as given below:

Case-I: $\quad X_{1: m: n}, \cdots, X_{2: m: n}, \cdots, X_{k: m: n}, \quad$ if $T<X_{k: m: n}$,

Case-II: $\quad X_{1: m: n}, \cdots, X_{k: m: n}, \cdots, X_{D: m: n}, \quad$ if $X_{k: m: n}<T<X_{m: m: n}$,

Case-III: $\quad X_{1: m: n}, \cdots, X_{k: m: n}, \cdots, X_{m: m: n}, \quad$ if $X_{k: m: n}<X_{m: m: n}<T$.

A pictorial representation of this censoring scheme is given in Figure 1. Note that for Case-I, $X_{k+1: m: n}, \cdots, X_{m: m: n}$ are not observed; likewise, for Case-II, $X_{D+1: m: n}, \cdots, X_{m: n: n}$ are not observed. The likelihood functions and log-likelihood function for \{Case-I, Case-II and CaseIII $\}$ denoted by $\left\{L_{I}, L_{I I}, \& L_{I I I}\right\}$ and $\left\{l_{I}, l_{I I}, \& l_{I I I}\right\}$, respectively are given below:

$$
\begin{aligned}
& \text { Case-I: } \quad L_{I}(\alpha, \beta)=K_{1} \prod_{j=1}^{k-1} f\left(x_{j: m: n}\right)\left[1-F\left(x_{j: m: n}\right)\right]^{R_{j}} f\left(x_{k: m: n}\right)\left[1-F\left(x_{k: m: n}\right)\right]^{R_{k}^{*}}, \\
& \text { Case-II: } L_{I I}(\alpha, \beta)=K_{2} \prod_{j=1}^{D} f\left(x_{j: m: n}\right)\left[1-F\left(x_{j: m: n}\right)\right]^{R_{j}}[1-F(T)]^{R_{D+1}^{*}}, \\
& \text { Case-III: } \quad L_{I I I}(\alpha, \beta)=K_{3} \prod_{j=1}^{m} f\left(x_{j: m: n}\right)\left[1-F\left(x_{j: m: n}\right)\right]^{R_{j}}
\end{aligned}
$$

where $K_{1}=\left[\prod_{j=1}^{k} \sum_{k=j}^{m}\left(R_{k}+1\right)\right], K_{2}=\left[\prod_{j=1}^{D} \sum_{k=j}^{m}\left(R_{k}+1\right)\right], K_{3}=\left[\prod_{j=1}^{m} \sum_{k=j}^{m}\left(R_{k}+\right.\right.$ 1)], $R_{k}^{*}=\left[n-k-\sum_{i=1}^{k-1} R_{i}\right]$ and $R_{D+1}^{*}=\left[n-D-\sum_{i=1}^{D} R_{i}\right]$. 
Number of removals

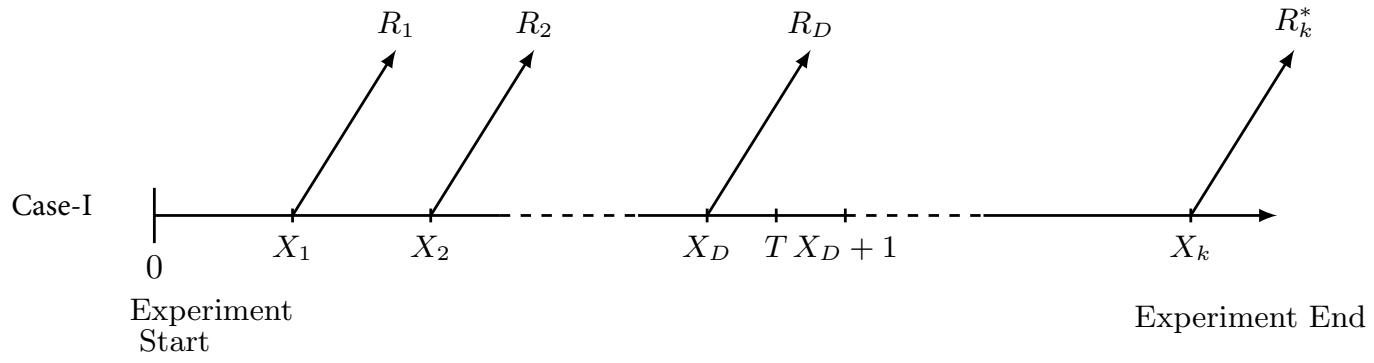

Number of removals

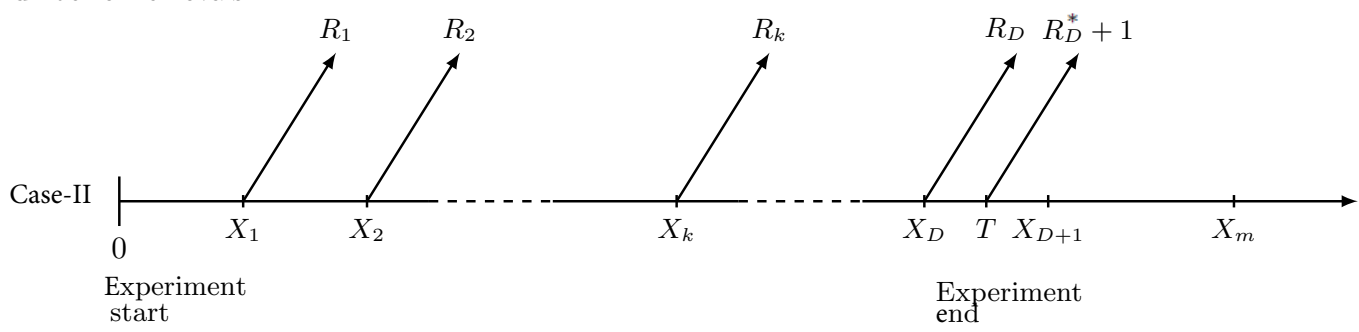

Number of removals

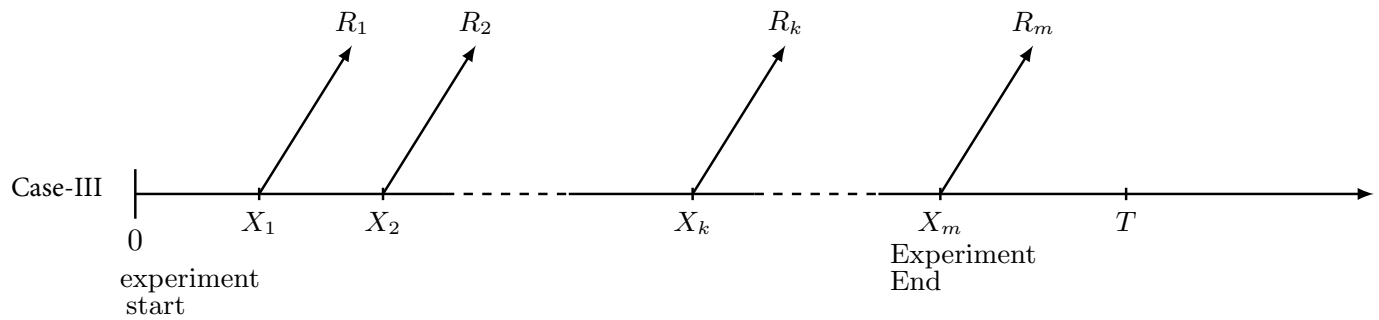

Figure 1: Schematic representation of generalised progressive hybrid censoring scheme

\section{Maximum likelihood estimation}

Maximum likelihood estimation is one of the most widely used methods under classical paradigm to obtain the estimator of the parameter of proposed distribution. In this section, we find the MLEs of $\alpha$ and $\beta$ of the considered distribution. The MLEs $\hat{\alpha}$ and $\hat{\beta}$ of $\alpha$ and $\beta$, respectively can be obtained by maximizing the likelihood function. Using the equations(1) and (2), the likelihood functions of $\alpha$ and $\beta$ are presented as follows:

Case-I: $L_{I} \propto(\alpha \beta)^{k}\left(1-e^{-\beta x_{k}}\right)^{\alpha-1} e^{-\beta x_{k}}\left[1-\left(1-e^{-\beta x_{k}}\right)^{\alpha}\right]^{R_{k^{*}}}$

$$
\prod_{j=1}^{k-1}\left(1-e^{-\beta x_{j}}\right)^{\alpha-1} e^{-\beta x_{j}}\left[1-\left(1-e^{-\beta x_{j}}\right)^{\alpha}\right]^{R_{j}},
$$

Case-II: $\quad L_{I I} \propto(\alpha \beta)^{D} \prod_{j=1}^{D}\left(1-e^{-\beta x_{j}}\right)^{\alpha-1} e^{-\beta x_{j}}\left[1-\left(1-e^{-\beta x_{j}}\right)^{\alpha}\right]^{R_{j}}$

$$
\left[1-\left(1-e^{-\beta T}\right)^{\alpha}\right]^{R_{D+1}^{*}}
$$

Case-III: $\quad L_{I I I} \propto(\alpha \beta)^{m} \prod_{j=1}^{m}\left(1-e^{-\beta x_{j}}\right)^{\alpha-1} e^{-\beta x_{j}}\left[1-\left(1-e^{-\beta x_{j}}\right)^{\alpha}\right]^{R_{j}}$.

The corresponding log-likelihood functions $\left\{l_{I}, l_{I I}, \& l_{I I I}\right\}$ are:

$$
\begin{aligned}
\text { Case-I: } & l_{I}(\alpha, \beta) \propto k \ln (\alpha \beta)+(\alpha-1) \sum_{j=1}^{k} \ln \left(1-e^{-\beta x_{j}}\right) \\
& +\sum_{j=1}^{k-1} R_{j} \ln \left[1-\left(1-e^{-\beta x_{j}}\right)^{\alpha}\right]+R_{k}^{*} \ln \left[1-\left(1-e^{-\beta x_{k}}\right)^{\alpha}\right]-\beta \sum_{j=1}^{k} x_{j},
\end{aligned}
$$




$$
\begin{aligned}
\text { Case-II: } & l_{I I}(\alpha, \beta) \propto D \ln (\alpha \beta)+(\alpha-1) \sum_{j=1}^{D} \ln \left(1-e^{-\beta x_{j}}\right) \\
& +\sum_{j=1}^{D} R_{j} \ln \left[1-\left(1-e^{-\beta x_{j}}\right)^{\alpha}\right]+R_{D+1}^{*} \ln \left[1-\left(1-e^{-\beta T}\right)^{\alpha}\right]-\beta \sum_{j=1}^{D} x_{j},
\end{aligned}
$$

Case-III: $l_{I I I}(\alpha, \beta) \propto m \ln (\alpha \beta)+(\alpha-1) \sum_{j=1}^{m} \ln \left(1-e^{-\beta x_{j}}\right)$

$$
+\sum_{j=1}^{m} R_{j} \ln \left[1-\left(1-e^{-\beta x_{j}}\right)^{\alpha}\right]-\beta \sum_{j=1}^{m} x_{j} .
$$

Furthermore, all three cases can be joined to give the combined likelihood function as:

$$
L(\alpha, \beta) \propto(\alpha \beta)^{J} \prod_{j=1}^{J}\left(1-e^{-\beta x_{j}}\right)^{\alpha-1} e^{-\beta x_{j}}\left[1-\left(1-e^{-\beta x_{j}}\right)^{\alpha}\right]^{R_{j}} \times W(\alpha, \beta),
$$

where $W(\alpha, \beta)= \begin{cases}1, & \text { if } J=k, m \\ {\left[1-\left(1-e^{-\beta T}\right)^{\alpha}\right]^{R_{D+1}^{*},},} & \text { if } J=D .\end{cases}$

and hence, the log-likelihood function will be

$$
\begin{aligned}
l(\alpha, \beta) \propto J \times & \ln (\alpha \beta)+(\alpha-1) \sum_{j=1}^{J} \ln \left(1-e^{-\beta x_{j}}\right) \\
& +\sum_{j=1}^{J} R_{j} \ln \left[1-\left(1-e^{-\beta x_{j}}\right)^{\alpha}\right]-\beta \sum_{j=1}^{J} x_{j}+\ln W(\alpha, \beta),
\end{aligned}
$$

Differentiating it with respect to the parameters $\alpha$ and $\beta$, respectively, we get:

$$
\begin{array}{r}
\frac{\partial l(\alpha, \beta)}{\partial \alpha}=\frac{J}{\alpha}+\sum_{j=1}^{J} \ln \left(1-e^{-\beta x_{j}}\right)-\sum_{j=1}^{J} \frac{R_{j}\left(1-e^{-\beta x_{j}}\right)^{\alpha} \ln \left(1-e^{-\beta x_{j}}\right)}{1-\left(1-e^{-\beta x_{j}}\right)^{\alpha}}+\frac{\partial \ln W(\alpha, \beta)}{\partial \alpha} \\
\frac{\partial l(\alpha, \beta)}{\partial \beta}=\frac{J}{\beta}+(\alpha-1) \sum_{j=1}^{J} \frac{x_{j} e^{-\beta x_{j}}}{\left(1-e^{-\beta x_{j}}\right)}-\sum_{j=1}^{J} \frac{R_{j} \alpha\left(1-e^{-\beta x_{j}}\right)^{\alpha-1} x_{j} e^{-\beta x_{j}}}{1-\left(1-e^{-\beta x_{j}}\right)^{\alpha}}-\sum_{j=1}^{J} x_{j}+\frac{\partial \ln W(\alpha, \beta)}{\partial \beta}
\end{array}
$$

where $\frac{\partial \ln W(\alpha, \beta)}{\partial \alpha}=-\frac{R_{D+1}^{*}\left(1-e^{-\beta T}\right)^{\alpha}}{1-\left(1-e^{-\beta T}\right)^{\alpha}} \times \ln \left(1-e^{-\beta T}\right)$ and $\frac{\partial \ln W(\alpha, \beta)}{\partial \beta}=-\frac{R_{D+1}^{*} \alpha\left(1-e^{-\beta T}\right)^{\alpha-1}}{1-\left(1-e^{-\beta T}\right)^{\alpha}} \times T e^{-\beta T}$, if $J=D$ and zero otherwise. The MLEs of $\alpha$ and $\beta$ can be obtained by solving likelihood equations (8) and (9) simultaneously. However, it may be noted that explicit solutions of the above equations are difficult to find. Therefore, this underscores the need for an alternative method, i.e., a numerical method to obtain the solution for the above two nonlinear equations. We have used the $\operatorname{optim}(\cdot)$ function, which is available in R-software, to maximize the logarithm of the likelihood function.

\section{Expected total time of the test}

In order to envision a successful compilation of a test plan, one should have significant knowledge of the expected total time of the test(ETTT). Therefore, the researchers are enthusiastically computing the ETTT to explore the experimental time duration for selecting an appropriate sampling plan. The ETTT is defined as the expected time required to complete the experiment. The cost and time duration to complete a test are interrelated. A significant high ETTT ultimately results into a high cost of conducting an experiment. Hence, impending need is to find the ETTT. In this section, the expressions for the ETTT under a GPH cesoring scheme are derived. The expected total time required to complete the experiment under GPH censoring with fixed number of removals is defined as:

$$
\begin{aligned}
E(T T T)= & T \times \operatorname{Pr}\left[X_{k: m: n}<T<X_{m: m: n}\right]+E\left(X_{m: m: n} \mid X_{m: m: n}<T\right) \\
& \times \operatorname{Pr}\left[X_{m: m: n}<T\right]+E\left[X_{k: m: n} \mid T<X_{k: m: n}\right] \operatorname{Pr}\left[T<X_{k: m: n}\right] .
\end{aligned}
$$


The expression for conditional expectation of $X_{j}$ given $R_{j}$ for progressive censoring scheme is given by Eissa et al. (2014) as:

$$
\begin{aligned}
E\left(X_{j} \mid R_{j}\right)=C(r) \sum_{S_{1}=0}^{r_{1}} \sum_{S_{2}=0}^{r_{2}} \sum_{S_{3}=0}^{r_{3}} \cdots & \sum_{S_{j}=0}^{r_{j}}(-1)^{A}\left(\begin{array}{c}
r_{1} \\
S_{1}
\end{array}\right)\left(\begin{array}{c}
r_{2} \\
S_{2}
\end{array}\right) \cdots\left(\begin{array}{c}
r_{j} \\
S_{j}
\end{array}\right) \\
& \times \frac{1}{\prod_{i=1}^{j-1} h\left(S_{i}\right)} \int_{0}^{\infty} t f(t) F^{B}(t) d t
\end{aligned}
$$

where $C(r)=\prod_{l=1}^{j}\left(n-l+1-\sum_{l_{1}=0}^{l-1} r_{l_{1}}\right), A=\sum_{i=1}^{j} S_{i}, h\left(S_{i}\right)=\sum_{i=1}^{j}\left(S_{i}+i\right)$ and $B=h\left(S_{j}\right)-1$. For our considered distribution, the expression comes out as

$$
\begin{aligned}
E\left(X_{j} \mid R_{j}\right)= & C(r) \sum_{S_{1}=0}^{r_{1}} \sum_{S_{2}=0}^{r_{2}} \sum_{S_{3}=0}^{r_{3}} \cdots \sum_{S_{j}=0}^{r_{j}}(-1)^{A}\left(\begin{array}{c}
r_{1} \\
S_{1}
\end{array}\right)\left(\begin{array}{c}
r_{2} \\
S_{2}
\end{array}\right) \cdots\left(\begin{array}{c}
r_{j} \\
S_{j}
\end{array}\right) \frac{1}{\prod_{i=1}^{j-1} h\left(S_{i}\right)} \\
& (\alpha \beta) \int_{0}^{\infty} t e^{-\beta t}\left(1-e^{-\beta t}\right)^{\alpha(1+B)-1} d t ; j=k, k+1, \cdots, m-1 \\
= & C(r) \sum_{S_{1}=0}^{r_{1}} \sum_{S_{2}=0}^{r_{2}} \sum_{S_{3}=0}^{r_{3}} \cdots \sum_{S_{j}=0}^{r_{j}}(-1)^{A}\left(\begin{array}{c}
r_{1} \\
S_{1}
\end{array}\right)\left(\begin{array}{c}
r_{2} \\
S_{2}
\end{array}\right) \cdots\left(\begin{array}{c}
r_{j} \\
S_{j}
\end{array}\right) \frac{1}{\prod_{i=1}^{j-1} h\left(S_{i}\right)} \\
& (\alpha \beta) \sum_{i=0}^{\infty}(-1)^{i}\left(\begin{array}{c}
\alpha(1+B)-1) \\
i
\end{array}\right) \frac{\Gamma 2}{(\beta(1+i))^{2}} ; j=k, k+1, \cdots, m-1
\end{aligned}
$$

Let $J$ denotes the number of observed failures upto termination time. For considered distribution, the probability mass function of the value $J$ for a prefixed value of $T$ is

$$
\begin{gathered}
\operatorname{Pr}[J=k]=\operatorname{Pr}\left[X_{k: m: n}>T\right]=\left[1-\left(1-e^{-\beta t}\right)^{\alpha}\right]^{n-k+1} \\
\operatorname{Pr}[J=m]=\operatorname{Pr}\left[X_{m: m: n}<T\right] \\
=c_{m-1} \sum_{i=1}^{m} \frac{a_{i, m}}{\gamma_{i}}\left[1-\left(1-\left(1-e^{-\beta T}\right)^{\alpha}\right)^{\gamma_{i}}\right] \\
\operatorname{Pr}\left[X_{k: m: n}<T<X_{m: m: n}\right]=\sum_{j=k}^{m-1} P\left(X_{j}<T<X_{j+1}\right) \\
P\left(X_{j}<T<X_{j+1}\right)=\left(1-\left(1-e^{-\beta T}\right)^{\alpha}\right)^{\gamma_{j+1}} c_{j-1} \\
\sum_{i^{\prime}=1}^{j} a_{i^{\prime} j}(\alpha \beta) \sum_{m_{1}=0}^{\infty}(-1)^{m_{1}}\left(\begin{array}{c}
\gamma_{i^{\prime}}-\gamma_{j}-2 \\
m_{1}
\end{array}\right) \\
\sum_{l=0}^{\infty}(-1)^{l}\left(\begin{array}{c}
\alpha m_{1}+\alpha-1 \\
l
\end{array}\right) \frac{1-e^{-T \beta(l+1)}}{\beta(l+1)} \\
; j=k, k+1, \cdots, m-1
\end{gathered}
$$

Where $\gamma_{j}=n-j+1+\sum_{i=j}^{m} R_{i} ; 1 \leq j \leq m, c_{j-1}=\prod_{i=1}^{j} \gamma_{i} ; 1 \leq j \leq m$, $a_{i, j}=\prod_{k \neq i=1}^{j} \frac{1}{\gamma_{k}-\gamma_{i}} ; 1 \leq i \leq j \leq m$; see Hemmati and Khorram (2013).

$$
f_{x_{k}}\left(x \mid X_{k: m: n}>T\right)=\frac{f_{X_{k}}(x)}{\operatorname{Pr}\left[X_{k: m: n}>T\right]}
$$




$$
\begin{aligned}
& f_{x_{m}}\left(x \mid X_{m: m: n}<T\right)=\frac{f_{X_{m}}(x)}{\operatorname{Pr}\left[X_{m: m: n}<T\right]} \\
& f_{X_{j^{*}}}(x)=c_{j^{*}-1} \sum_{i=1}^{j^{*}} a_{i, j^{*}}(\alpha \beta) \sum_{k_{1}=0}^{\infty}(-1)^{k_{1}}\left(\begin{array}{c}
\gamma_{i}-1 \\
k_{1}
\end{array}\right) \sum_{k_{2}=0}^{\infty}(-1)^{k_{2}}\left(\begin{array}{c}
\alpha k_{1}+\alpha-1 \\
k_{2}
\end{array}\right) \\
& \left(e^{-\beta x}\right)^{\left(k_{2}+1\right)} ; j^{*}=k, m \\
& E\left(X_{m} \mid X_{m: m: n}<T\right)=\int_{0}^{T} x f_{x_{m}}\left(x \mid X_{m: m: n}<T\right) d x \\
& =\frac{c_{m-1}}{\operatorname{Pr}\left[X_{m: m: n}<T\right]} \sum_{i=1}^{m} a_{i, m}(\alpha \beta) \sum_{k_{1}=0}^{\infty}(-1)^{k_{1}}\left(\begin{array}{c}
\gamma_{i}-1 \\
k_{1}
\end{array}\right) \\
& \sum_{k_{2}=0}^{\infty}(-1)^{k_{2}}\left(\begin{array}{c}
\alpha k_{1}+\alpha-1 \\
k_{2}
\end{array}\right) \\
& \frac{1}{\beta\left(k_{2}+1\right)}\left[\frac{1}{\beta\left(k_{2}+1\right)}-\frac{e^{-\beta T\left(k_{2}+1\right)}}{\beta\left(k_{2}+1\right)}-T e^{-\beta T\left(k_{2}+1\right)}\right] ; \\
& E\left(X_{k} \mid X_{k: m: n}>T\right)=\int_{T}^{\infty} x f_{x_{k}}\left(x \mid X_{k: m: n}>T\right) d x \\
& =\frac{c_{k-1}}{\operatorname{Pr}\left[X_{k: m: n}>T\right]} \sum_{i=1}^{k} a_{i, k}(\alpha \beta) \sum_{k_{1}=0}^{\infty}(-1)^{k_{1}}\left(\begin{array}{c}
\gamma_{i}-1 \\
k_{1}
\end{array}\right) \\
& \sum_{k_{2}=0}^{\infty}(-1)^{k_{2}}\left(\begin{array}{c}
\alpha k_{1}+\alpha-1 \\
k_{2}
\end{array}\right) \\
& \frac{1}{\beta\left(k_{2}+1\right)}\left[\frac{e^{-\beta T\left(k_{2}+1\right)}}{\beta\left(k_{2}+1\right)}+T e^{-\beta T\left(k_{2}+1\right)}\right] ;
\end{aligned}
$$

The equations (12), (13), (14), (15) and (16) can be put simultaneously in equation (10) to get the final expression for the ETTT as

$$
\begin{aligned}
E(T T T)= & \sum_{j=k}^{m-1}\left(1-\left(1-e^{-\beta T}\right)^{\alpha}\right)^{\gamma_{j+1}} c_{j-1} \sum_{i^{\prime}=1}^{j} a_{i^{\prime} j}(\alpha \beta) \sum_{m_{1}=0}^{\infty}(-1)^{m_{1}}\left(\begin{array}{c}
\gamma_{i^{\prime}}-\gamma_{j}-2 \\
m_{1}
\end{array}\right) \\
& \times \sum_{l=0}^{\infty}(-1)^{l}\left(\begin{array}{c}
\alpha m_{1}+\alpha-1 \\
l
\end{array}\right) \frac{1-e^{-T \beta(l+1)}}{\beta(l+1)} \\
& +c_{m-1} \sum_{i=1}^{m} \frac{a_{i, m}}{\gamma_{i}}\left[1-\left(1-\left(1-e^{-\beta T}\right)^{\alpha}\right)^{\gamma_{i}}\right] \frac{c_{m-1}}{\operatorname{Pr}\left[X_{m: m: n}<T\right]} \sum_{i=1}^{m} a_{i, m}(\alpha \beta) \sum_{k_{1}=0}^{\infty}(-1)^{k_{1}}\left(\begin{array}{c}
\gamma_{i}-1 \\
k_{1}
\end{array}\right) \\
& \times \sum_{k_{2}=0}^{\infty}(-1)^{k_{2}}\left(\begin{array}{c}
\alpha k_{1}+\alpha-1 \\
k_{2}
\end{array}\right) \frac{1}{\beta\left(k_{2}+1\right)}\left[\frac{1}{\beta\left(k_{2}+1\right)}-\frac{e^{-\beta T\left(k_{2}+1\right)}}{\beta\left(k_{2}+1\right)}-T e^{-\beta T\left(k_{2}+1\right)}\right] \\
& +\left[1-\left(1-e^{-\beta t}\right)^{\alpha}\right]^{n-k+1} \frac{c_{k-1}}{\operatorname{Pr}\left[X_{k: m: n}>T\right]} \sum_{i=1}^{k} a_{i, k}(\alpha \beta) \sum_{k_{1}=0}^{\infty}(-1)^{k_{1}}\left(\begin{array}{c}
\gamma_{i}-1 \\
k_{1}
\end{array}\right) \\
& \times \sum_{k_{2}=0}^{\infty}(-1)^{k_{2}}\left(\begin{array}{c}
\alpha k_{1}+\alpha-1 \\
k_{2}
\end{array}\right) \frac{1}{\beta\left(k_{2}+1\right)}\left[\frac{e^{-\beta T\left(k_{2}+1\right)}}{\beta\left(k_{2}+1\right)}+T e^{-\beta T\left(k_{2}+1\right)}\right] .
\end{aligned}
$$

\subsection{Expected number of failures (ETNF)}

A corollary to ETTT is ETNFs that can also be used to provide an action plan to execute an experiment. Contrary to ETTT, ETNFs ensures the efficiency of the test conducted. 
A significant large number of failures which occur during the experiment result into high efficiency but add to the cost of experimentation. Owing to its practical utility, this section is devoted to its derivation. The expected number of failures based on a GPH censoring scheme is given by

$$
\begin{aligned}
E\left(M_{G P H}\right) & =\sum_{j=k}^{m} j \times P[J=j] \\
& =k\left[1-\left(1-e^{-\beta t}\right)^{\alpha}\right]^{n-k+1}+m\left[c_{m-1} \sum_{i=1}^{m} \frac{a_{i, m}}{\gamma_{i}}\left(1-\left(1-\left(1-e^{-\beta T}\right)^{\alpha}\right)^{\gamma_{i}}\right)\right] \\
+ & \sum_{j=k}^{m-1} j\left(1-\left(1-e^{-\beta T}\right)^{\alpha}\right)^{\gamma_{j+1}} c_{j-1} \sum_{i^{\prime}=1}^{j} a_{i^{\prime} j}(\alpha \beta) \sum_{m_{1}=0}^{\infty}(-1)^{m_{1}}\left(\begin{array}{c}
\gamma_{i^{\prime}}-\gamma_{j}-2 \\
m_{1}
\end{array}\right) \\
& \sum_{l=0}^{\infty}(-1)^{l}\left(\begin{array}{c}
\alpha m_{1}+\alpha-1 \\
l
\end{array}\right) \frac{1-e^{-T \beta(l+1)}}{\beta(l+1)}
\end{aligned}
$$

\section{Entropy estimation in case of the GPH censoring scheme}

The entropy as a measure of uncertainty covered by model $f(\cdot)$ having $\operatorname{cdf} F(\cdot)$, pioneered by Shannon (1948), is defined as

$$
H(X)=-\int_{-\infty}^{\infty} \log f(x) d F(x)
$$

Consider a life testing experiment with $n$ units. The units are to be observed until its failure. Suppose the lifetime of $\mathrm{n}$ units are independently and identically distributed with $\operatorname{cdf} F(\cdot)$ and pdf $f(\cdot)$. But owing to some constraints, lifetimes of all $\mathrm{n}$ items are not observed and censored data are available to study. Thus, a natural question which strikes the mind of researcher is whether the censored sample contain the same or less information as compared to complete sample. Figuratively speaking, the entropy measure provides us a tool for measuring the loss of information due to censoring. Following Kaushik et al. (2016), the joint distribution of observed sample under considered censoring scheme can be written as:

$$
\begin{aligned}
P\left[x_{1: m: n},\right. & \left.x_{2: m: n}, \cdots, x_{j: m: n}, R_{1}, R_{2}, \cdots, R_{j}, J=j\right] \\
& =L\left(x_{1: m: n}, x_{2: m: n}, \cdots, x_{j: m: n} \mid R_{1}, R_{2}, \cdots, R_{j}, J=j\right) * P[J=j] \\
& =f^{*}(x)(\text { say })
\end{aligned}
$$

and hence, Shannon entropy of observed censored sample will be

$$
E\left(-\log f^{*}(x)\right)=\int_{\widetilde{x}}-\log \left(f^{*}(x)\right) d F^{*}(x),
$$

where $L(\cdot)$ is given in equation(6) and the probabilities $P[J=k], P[J=j]$ and $P[J=m]$ are given in equations(12), (13) and (14), respectively. The obtained expression for Shannon entropy is too complicated to write in closed form. Hence, we propose the use of following simple algorithm for its computation, which is developed following Kaushik et al. (2016).

\section{Algorithm for computation of entropy:}

1. Set $i=1$,

2. Generate a GPH sample $\underset{\sim}{x}$ from EED using the algorithm given in Section 7 .

3. Compute $\left.H_{i}=-\log \left(f^{*} \underset{\sim}{x}\right)\right)$ and set $i=i+1$. 
4. Repeat step 2 to 3 till $i \leq N$, where $N$ is a sufficiently large number.

5. Compute $\mathrm{H}=\frac{1}{N} \sum_{i=1}^{N} H_{i}$, which is the approximated value of Shannon entropy.

Furthermore, Shannon entropy in case of complete sample for considered model given in equation (1) will be,

$$
\begin{aligned}
H_{c} & =-\int \log f(x) d F(x) \\
& =-\log \alpha-\log \beta+(\Psi(\alpha+1)-\psi(1))+\frac{\alpha-1}{\alpha},
\end{aligned}
$$

where, $\psi($.$) denotes the Digamma function.$

\subsection{Awad sub-entropy measures for GPH censoring scheme}

Awad and Alawneh (1987) modified the earlier entropy measure, which was given by Shannon (1948). The motivation for considering the new measure was to combat the deficiency of Shannon measure which sometimes give the negative value as in the case of continuous distribution. Awad Sub-entropy is defined as

$$
H_{\text {Awad }}=-E\left(\ln \frac{f(x)}{\delta}\right)
$$

where $\delta=\sup _{x} f(x)$.

For our considered model i.e., EED in equation(1), we have,

$$
\begin{gathered}
\ln f(x, \alpha, \beta)=\ln \alpha+\ln \beta-\beta x+(\alpha-1) \ln \left(1-e^{-\beta x}\right) \\
\frac{\partial \log f(x, \alpha, \beta)}{\partial x}=-\beta+\frac{(\alpha-1) \beta e^{-\beta x}}{1-e^{-\beta x}}=0
\end{gathered}
$$

we get, $x=\frac{\ln (\alpha)}{\beta}$. We put this value of $x$ in $f(x)$, we have

$$
\sup _{x} f(x)=\beta\left(1-\frac{1}{\alpha}\right)^{\alpha-1} ; \alpha>1
$$

Since the expression for Awad Sub-entropy is too complicated to write in closed form. Hence, the same algorithm which is given in Section 5 to compute Shannon entropy, can be used here too.

\section{Real data illustration}

We fitted our considered model on a various real dataset. The considered dataset represents the waiting times (in minutes) before service of 100 bank customers as reported and analyzed by Ghitany et al. (2008). The dataset consists of the following 100 observations:

$0.8,0.8,1.3,1.5,1.8,1.9,1.9,2.1,2.6,2.7,2.9,3.1,3.2,3.3,3.5,3.6,4,4.1,4.2,4.2,4.3,4.3$, $4.4,4.4,4.6,4.7,4.7,4.8,4.9,4.9,5,5.3,5.5,5.7,5.7,6.1,6.2,6.2,6.2,6.3,6.7,6.9,7.1,7.1$, $7.1,7.1,7.4,7.6,7.7,8,8.2,8.6,8.6,8.6,8.8,8.8,8.9,8.9,9.5,9.6,9.7,9.8,10.7,10.9,11,11$, $11.1,11.2,11.2,11.5,11.9,12.4,12.5,12.9,13,13.1,13.3,13.6,13.7,13.9,14.1,15.4,15.4$, $17.3,17.3,18.1,18.2,18.4,18.9,19,19.9,20.6,21.3,21.4,21.9,23,27,31.6,33.1,38.5$.

The fitting summary along with the MLEs of the parameters $\alpha$ and $\beta$ denoted as $\hat{\alpha}_{M L}$ and $\hat{\beta}_{M L}$, respectively and the standard errors(SEs) are given below: 


\begin{tabular}{lllll}
\hline p-value & K-S distance & loglikelihood & AIC & BIC \\
0.9969 & 0.0403 & -317.0953 & 638.1906 & 643.4009 \\
& & & & \\
$\hat{\alpha}_{M L}$ & $S E\left(\hat{\alpha}_{M L}\right)$ & $\hat{\beta}_{M L}$ & $S E\left(\hat{\beta}_{M L}\right)$ & \\
2.1832 & 0.3343 & 0.1591 & 0.0175 & \\
\hline
\end{tabular}

The above summary indicates that EED fits well to the data. For the illustration of our methodology, from the considered data, we have generated censored data for a prefixed $m, k$, $T$, and number of removals. We have considered different removal patterns by fixing values of $R_{1}, R_{2}, \cdots, R_{m}$ for a set of values of $m, k$ and $T$. The schemes have been considered are as follows:

$S_{m: n}{ }^{(1)}:$ All the removals are at the last failure, i.e., $R_{m}=n-m$.

$S_{m: n}{ }^{(2)}$ : All the removals are at the first failure, i.e., $R_{1}=n-m$.

$S_{m: n}{ }^{(3)}$ : The removals are at the first and last failure, i.e., $R_{1}=R_{m}=(n-m) / 2$.

$S_{m: n}{ }^{(4)}$ : The removals are at middle failure, i.e., $R_{m / 2}=R_{m / 2+1}=(n-m) / 2$.

We set the values of $m, k$ and $T$ to get the censored data for finding the MLEs and asymptotic confidence intervals(ACIs). Since the likelihood equations do not have an explicit solution, the numerical method coupled with $\mathrm{R}$ software is maneuvered. Here, the optim(.) function is used to find the ML estimates of the parameters. Using the concept of the large sample theory, the asymptotic confidence intervals for $\alpha$ and $\beta$ are also computed as

$$
A C I_{\alpha}=\left[\hat{\alpha}_{M L} \pm 1.96 \sqrt{V\left(\hat{\alpha}_{M L}\right)}\right] \quad \& \quad A C I_{\beta}=\left[\hat{\beta}_{M L} \pm 1.96 \sqrt{V\left(\hat{\beta}_{M L}\right)}\right]
$$

respectively. Here, $V(\cdot)$ denotes the asymptotic variance, and it is computed by finding the inverse of the estimated Fisher information matrix. Table 1 enlists the MLEs, the estimated 95\% asymptotic confidence intervals, the estimated Shannon entropies and the estimated Awad sub-entropies under the GPH censoring scheme for all four removal patterns and the different choices of $m, k$ and $T$ for the considered real data of waiting times. From Table 1, one can easily conclude that as the value of $m, k$ and $T$ decreases (increases), the estimated Shannon entropies decrease (increase) and the length of ACIs increase(decrease). Furthermore, the estimated Shannon entropies and Awad sub-entropies are lowest for $S_{m: n}{ }^{(1)}$ and highest for $S_{m: n}{ }^{(4)}$. Both entropies for $S_{m: n}{ }^{(3)}$ are higher than the corresponding entropies for $S_{m: n}{ }^{(2)}$. It may be noted here that the estimates obtained under various censoring schemes are close to those obtained from the complete sample, although variations can be seen from one scheme to other.

\section{Simulation study}

Here, a simulation study is conducted to examine the performance of the estimates of the parameters $\alpha$ and $\beta$ based on mean squared errors(MSEs) under the considered censoring scheme. It is important to note that the exact expressions for the MSEs cannot be obtained because the estimators are not found in explicit form. Therefore, the MSEs of the estimators are estimated based on a simulation study of 10,000 samples. It may be noted here that the MSEs of the estimators will depend on values of $n, k, m, T, \alpha$ and $\beta$, and hence, various choices have been made to study the effect thereof. We are proposing the following algorithm to generate the sample under the considered censoring scheme:

\section{Algorithm: Simulation of GPH Censored Data}

1. Generate $k$ independent standard uniform random variables $U_{1}, U_{2}, \cdots, U_{k}$.

2. Compute $B_{i}=U^{1 / \gamma_{i}} ; i=1, \cdots, k$, where $\gamma_{i}=n-i+1+\sum_{j=i}^{m} R_{j}$. 
Table 1: The MLEs of the parameters, the estimated 95\% ACIs, the estimated Shannon entropies and the estimated Awad sub-entropies under the GPH censoring scheme for considered real data set.

\begin{tabular}{|c|c|c|c|c|c|c|c|}
\hline $\mathrm{m}$ & $\mathrm{k}$ & $\mathrm{T}$ & $\mathrm{R}$ & $\hat{\alpha}_{M L}(95 \% \mathrm{ACI})$ & $\hat{\beta}_{M L}(95 \% \mathrm{ACI})$ & $\hat{H}$ & $\hat{H}_{a w a d}$ \\
\hline \multirow[t]{4}{*}{80} & 20 & 20 & $S_{m: n}{ }^{(1)}$ & $2.3041(2.2288,2.3794)$ & $0.1685(0.1644,0.1726)$ & 3.1255 & 38.9631 \\
\hline & & & $S_{m: n}^{(2)}$ & $2.4367(2.3529,2.5206)$ & $0.1643(0.1602,0.1683)$ & 3.1444 & 40.1485 \\
\hline & & & $S_{m: n}(3)$ & $2.3401(2.2619,2.4182)$ & $0.1644(0.1605,0.1684)$ & 3.1538 & 40.4796 \\
\hline & & & $S_{m: n}{ }^{(4)}$ & $2.0545(1.9873,2.1218)$ & $0.1482(0.1442,0.1521)$ & 3.2240 & 43.9639 \\
\hline \multirow[t]{4}{*}{80} & 20 & 16 & $S_{m: n}{ }^{(1)}$ & $2.3072(2.2317,2.3826)$ & $0.1686(0.1644,0.1727)$ & 3.1195 & 38.7704 \\
\hline & & & $S_{m: n}(2)$ & $2.5537(2.4628,2.6446)$ & $0.1737(0.1692,0.1781)$ & 3.1207 & 38.8773 \\
\hline & & & $S_{m: n}(3)$ & $2.4106(2.3284,2.4927)$ & $0.1683(0.1642,0.1725)$ & 3.1374 & 39.6879 \\
\hline & & & $S_{m: n}{ }^{(4)}$ & $2.1107(2.0387,2.1827)$ & $0.1533(0.1492,0.1576)$ & 3.1847 & 40.5632 \\
\hline \multirow[t]{4}{*}{80} & 40 & 20 & $S_{m: n}{ }^{(1)}$ & $2.3157(2.2397,2.3917)$ & $0.1686(0.1644,0.1727)$ & 3.1264 & 39.0206 \\
\hline & & & $S_{m: n}{ }^{(2)}$ & $2.2271(2.1527,2.3015)$ & $0.1605(0.1565,0.1645)$ & 3.1638 & 40.8808 \\
\hline & & & $S_{m: n}{ }^{(3)}$ & $2.2884(2.2134,2.3634)$ & $0.1622(0.1583,0.1661)$ & 3.1653 & 40.9941 \\
\hline & & & $S_{m: n}{ }^{(4)}$ & $2.0476(1.9802,2.1149)$ & $0.1476(0.1436,0.1516)$ & 3.2271 & 44.1249 \\
\hline \multirow[t]{4}{*}{80} & 40 & 16 & $S_{m: n}{ }^{(1)}$ & $2.3275(2.2512,2.4038)$ & $0.1696(0.1655,0.1738)$ & 3.1253 & 38.9716 \\
\hline & & & $S_{m: n}^{(2)}$ & $2.0977(2.0262,2.1694)$ & $0.1624(0.1582,0.1668)$ & 3.1381 & 39.3361 \\
\hline & & & $S_{m: n}(3)$ & $2.0812(2.0122,2.1502)$ & $0.1558(0.1517,0.1598)$ & 3.1576 & 40.4125 \\
\hline & & & $S_{m: n}(4)$ & $2.3108(2.2314,2.3903)$ & $0.1686(0.1641,0.1732)$ & 3.1857 & 40.9767 \\
\hline \multirow[t]{4}{*}{60} & 20 & 20 & $S_{m: n}{ }^{(1)}$ & $2.2774(2.1941,2.3607)$ & $0.1673(0.1622,0.1723)$ & 3.1047 & 38.1885 \\
\hline & & & $S_{m: n}{ }^{(2)}$ & $2.5718(2.4725,2.6711)$ & $0.1695(0.1648,0.1742)$ & 3.1108 & 38.8675 \\
\hline & & & $S_{m: n}(3)$ & $2.0695(1.9964,2.1426)$ & $0.1529(0.1484,0.1573)$ & 3.1206 & 39.1071 \\
\hline & & & $S_{m: n}(4)$ & $2.5407(2.4496,2.6318)$ & $0.1911(0.1856,0.1966)$ & 3.1423 & 40.1341 \\
\hline \multirow[t]{4}{*}{60} & 20 & 16 & $S_{m: n}{ }^{(1)}$ & $2.3129(2.2280,2.3977)$ & $0.1693(0.1642,0.1744)$ & 3.1003 & 38.1456 \\
\hline & & & $S_{m: n}(2)$ & $2.5003(2.4014,2.5993)$ & $0.1718(0.1667,0.1769)$ & 3.1045 & 38.1885 \\
\hline & & & $S_{m: n}{ }^{(3)}$ & $2.2660(2.1849,2.3471)$ & $0.1588(0.1544,0.1633)$ & 3.1105 & 38.7701 \\
\hline & & & $S_{m: n}{ }^{(4)}$ & $2.2805(2.1962,2.3649)$ & $0.1706(0.1651,0.1763)$ & 3.1108 & 39.0403 \\
\hline \multirow[t]{4}{*}{60} & 40 & 20 & $S_{m: n}{ }^{(1)}$ & $2.0762(2.0013,2.1512)$ & $0.1445(0.1403,0.1487)$ & 3.1218 & 38.2667 \\
\hline & & & $S_{m: n}^{(2)}$ & $2.2959(2.2116,2.3802)$ & $0.1618(0.1630,0.1731)$ & 3.1259 & 39.0138 \\
\hline & & & $S_{m: n}{ }^{(3)}$ & $2.5762(2.4798,2.6725)$ & $0.1729(0.1681,0.1776)$ & 3.1476 & 39.2222 \\
\hline & & & $S_{m: n}{ }^{(4)}$ & $2.4359(2.3488,2.5232)$ & $0.1807(0.1754,0.1862)$ & 3.1688 & 40.2381 \\
\hline \multirow[t]{4}{*}{60} & 40 & 16 & $S_{m: n}{ }^{(1)}$ & $2.3038(2.2193,2.3884)$ & $0.1686(0.1636,0.1737)$ & 3.1087 & 38.1974 \\
\hline & & & $S_{m: n}(2)$ & $2.2991(2.2105,2.3877)$ & $0.1691(0.1640,0.1742)$ & 3.1150 & 38.7592 \\
\hline & & & $S_{m: n}(3)$ & $2.1478(2.0711,2.2246)$ & $0.1585(0.1539,0.1631)$ & 3.1217 & 38.9332 \\
\hline & & & $S_{m: n}(4)$ & $2.6717(2.5724,2.7709)$ & $0.2026(0.1967,0.2086)$ & 3.1649 & 39.0408 \\
\hline
\end{tabular}


3. Start with $V_{0}=1$ and compute $V_{i}=B_{i} V_{i-1}$ and $U_{r: m: n}=1-V_{r} \forall r=1, \cdots, k$.

4. If $U_{k: m: n}>F(T)$, then Goto Step-6, Else $l=k+1$ and Goto Step-5.

5. While $V_{l-1}>1-F(T)$ and $l \leq m$ Do:

(a) Generate a uniform random variable U.

(b) Define $B_{l}=U^{\frac{1}{\sum_{i=l}^{m} R_{i}+1}}$ and $V_{l}=V_{l-1} * B_{l}$.

(c) $\mathrm{l}=\mathrm{l}+1$.

6. $X_{j}^{(I I)}=F^{\leftarrow}\left(1-V_{j}\right) ; j=1, \cdots, l$, and Stop.

Here $F^{\leftarrow}(\cdot)$ is the inverse CDF function defined as $F^{\leftarrow}(p)=\inf \{x \in R: F(x) \geq p\}$. Table 2 contains the results obtained through the simulation study for the GPH censored sample, considering only one removal pattern for various values of $n, m, k$ ( $T$ fixed) to observe the effect of these on entropy. In Table 2, three choices of $n$, i.e., $n=30$ (small), 50 (moderate), 100 (large) are considered. The value of $k$ and $m$ are set to be $50 \%$ and $72 \%$ of $n$, respectively. The removal pattern is taken as $S_{m: n}{ }^{(4)}$ i.e., $R_{m / 2}=R_{m / 2+1}=(n-m) / 2$. Table 2 also contains MSEs of the parameters denoted by $M S E\left(\hat{\alpha}_{M L}\right)$ and $M S E\left(\hat{\beta}_{M L}\right)$, the estimated $95 \%$ asymptotic confidence intervals, the estimated Shannon entropies and Awad sub-entropies. It is worthwhile to mention that for considered distribution with $\alpha<1$, the Awad sub-entropy does not exist; hence; the results are reported for $\alpha \geq 1$. From Table 2, we can conclude that for fixed $\alpha$, as $\beta$ increases, the MSEs of $\beta$ increase. Similarly, for fixed $\beta$, as $\alpha$ increases, the MSEs of $\alpha$ increase. For fixed $\alpha$ and $\beta$, as $n$ increases(for fixed $k, m$ and $T$ ), the MSEs of both the parameters decrease. The estimated Shannon entropies for fixed $\alpha$ and $\beta$ generally increase as $n$ increases for fixed $k, m$, and $T$. For some of the values of $\alpha$ and $\beta$, the estimated Shannon entropies in the censored case show the negative value.

Table 2: The MLEs of the parameters along with the MSEs, the estimated 95\% ACIs, the estimated Shannon entropies and the estimated Awad sub-entropies based on simulation study for various censoring schemes considering removal pattern $S_{m: n}{ }^{(4)}$

\begin{tabular}{|c|c|c|c|c|c|c|c|c|c|c|}
\hline Censoring Scheme & $\alpha$ & $\beta$ & $\hat{\alpha}_{M L}$ & $M S E\left(\hat{\alpha}_{M L}\right)$ & ACI for $\alpha$ & $\hat{\beta}_{M L}$ & $M S E\left(\dot{\beta}_{M L}\right)$ & ACI for $\beta$ & $\hat{H}$ & $\hat{H}_{\text {Awad }}$ \\
\hline \multirow{10}{*}{$\begin{array}{c}\mathrm{n}=30, \mathrm{k}=15, \mathrm{~m}=22, \mathrm{~T}=10 \\
\mathrm{R}=\left(0^{*} 10,4^{*} 2,0^{*} 10\right)\end{array}$} & & & & & & & & & & \\
\hline & 0.5 & 0.5 & 0.5383 & 0.0107 & $(0.3177,0.8051)$ & 0.5576 & 0.0279 & $(0.1750,0.9743)$ & 0.9946 & \\
\hline & & 1 & 0.5399 & 0.0108 & $(0.2625,0.8520)$ & 1.1445 & 0.1272 & $(0.3077,2.0081)$ & 0.2796 & \\
\hline & & 2 & 0.5371 & 0.0114 & $(0.2391,0.9261)$ & 2.3082 & 0.5348 & $(0.3552,3.1962)$ & -0.4292 & \\
\hline & 1 & 0.5 & 1.0899 & 0.0563 & $(0.4307,1.4111)$ & 0.5521 & 0.0181 & $(0.1950,0.8519)$ & 1.6506 & 3.7558 \\
\hline & & 1 & 1.0813 & 0.0573 & $(0.3952,1.5219)$ & 1.0961 & 0.0738 & $(0.2275,2.4972)$ & 0.9565 & 1.0771 \\
\hline & & 2 & 1.1078 & 0.0637 & $(0.2946,1.5731)$ & 2.2036 & 0.3111 & $(0.2958,2.9824)$ & 0.2721 & 0.1587 \\
\hline & 2 & 0.5 & 2.2165 & 0.2956 & $(0.9335,2.6437)$ & 0.5394 & 0.0126 & $(0.2649,0.8944)$ & 1.9523 & 7.5093 \\
\hline & & 1 & 2.1942 & 0.3163 & $(0.7759,2.9151)$ & 1.0731 & 0.0511 & $(0.4151,1.5327)$ & 1.2619 & 2.4318 \\
\hline & & 2 & 2.2181 & 0.3613 & $(0.6868,3.1804)$ & 2.1462 & 0.2035 & $(0.8680,2.9058)$ & 0.5715 & 0.5526 \\
\hline \multirow{10}{*}{$\begin{array}{c}\mathrm{n}=50, \mathrm{k}=25, \mathrm{~m}=36, \mathrm{~T}=10 \\
\mathrm{R}=\left(0^{*} 17,7^{*} 2,0^{*} 17\right)\end{array}$} & & & & & & & & & & \\
\hline & 0.5 & 0.5 & 0.5141 & 0.0058 & $(0.3803,0.7506)$ & 0.5408 & 0.0214 & $(0.1939,0.9566)$ & 0.9617 & \\
\hline & & 1 & 0.5261 & 0.0067 & $(0.4005,0.8091)$ & 1.0971 & 0.0798 & $(0.3605,1.6361)$ & 0.2866 & \\
\hline & & 2 & 0.5251 & 0.0068 & $(0.4181,0.8673)$ & 2.1783 & 0.3341 & $(0.5477,2.9520)$ & -0.4021 & \\
\hline & 1 & 0.5 & 1.0564 & 0.0362 & $(0.6501,1.3061)$ & 0.5347 & 0.0116 & $(0.2904,0.8181)$ & 1.6603 & 3.6631 \\
\hline & & 1 & 1.0635 & 0.0363 & $(0.5422,1.3718)$ & 1.0722 & 0.0473 & $(0.4906,2.4695)$ & 0.9686 & 1.0804 \\
\hline & & 2 & 1.0691 & 0.0382 & $(0.4307,1.4445)$ & 2.1502 & 0.1845 & $(0.7102,2.7564)$ & 0.2759 & 0.1550 \\
\hline & 2 & 0.5 & 2.1955 & 0.1615 & $(1.6252,2.5293)$ & 0.5339 & 0.0082 & $(0.2837,0.6926)$ & 1.9600 & 7.5920 \\
\hline & & 1 & 2.1346 & 0.1694 & $(1.4588,2.8245)$ & 1.0372 & 0.0269 & $(0.5699,1.2706)$ & 1.2885 & 2.5448 \\
\hline & & 2 & 2.1463 & 0.175 & $(1.3083,3.0440)$ & 2.1209 & 0.1131 & $(1.4884,2.6788)$ & 0.5747 & 0.5561 \\
\hline \multirow{10}{*}{$\begin{array}{c}\mathrm{n}=100, \mathrm{k}=50, \mathrm{~m}=72, \mathrm{~T}=10 \\
\mathrm{R}=\left(0^{*} 35,14^{*} 2,0^{*} 35\right)\end{array}$} & & & & & & & & & & \\
\hline & 0.5 & 0.5 & 0.5102 & 0.0027 & $(0.4281,0.6305)$ & 0.5204 & 0.0081 & $(0.2795,0.6976)$ & 0.9893 & \\
\hline & & 1 & 0.5061 & 0.0028 & $(0.4171,0.6540)$ & 1.0352 & 0.0336 & $(0.5746,1.4097)$ & 0.2901 & \\
\hline & & 2 & 0.5112 & 0.0028 & $(0.4233,0.6631)$ & 2.0888 & 0.1203 & $(1.4236,2.8101)$ & -0.3982 & \\
\hline & 1 & 0.5 & 1.0293 & 0.0144 & $(0.8828,1.2510)$ & 0.5124 & 0.0045 & $(0.3366,0.6610)$ & 1.6868 & 3.6509 \\
\hline & & 1 & 1.0322 & 0.0151 & $(0.7975,1.3467)$ & 1.0391 & 0.0203 & $(0.6891,1.2473)$ & 0.9817 & 1.0564 \\
\hline & & 2 & 1.0226 & 0.0158 & $(0.7412,1.4185)$ & 2.0484 & 0.0774 & $(1.4574,2.6569)$ & 0.2972 & 0.1581 \\
\hline & 2 & 0.5 & 2.0518 & 0.0781 & $(1.6328,2.4194)$ & 0.5113 & 0.0032 & $(0.3195,0.5904)$ & 1.9850 & 7.8399 \\
\hline & & 1 & 2.0871 & 0.0799 & $(1.4982,2.6875)$ & 1.0292 & 0.0143 & $(0.5879,1.1312)$ & 1.2901 & 2.5472 \\
\hline & & 2 & 2.0803 & 0.0807 & $(1.4405,2.7824)$ & 2.0502 & 0.0527 & $(1.6586,2.5417)$ & 0.6001 & 0.5941 \\
\hline
\end{tabular}

Table 3 shows the MLEs, the MSEs, and the estimated 95\% asymptotic confidence intervals under the GPH censoring scheme for all four removal patterns taking $n=100$ and $T=10$. For a small number of removals, i.e., for large $\mathrm{m}$, the MSEs of both the parameters are lesser for the removal pattern $S_{m: n}{ }^{(2)}$ compared to $S_{m: n}{ }^{(1)}$, and the MSEs under $S_{m: n}{ }^{(3)}$ are observed to be less than that for $S_{m: n}{ }^{(4)}$. For large number of removals, the MSEs of both the parameters are lesser under the removal pattern $S_{m: n}{ }^{(1)}$ compared to $S_{m: n}{ }^{(2)}$, and the MSEs 
Table 3: The MLEs, the MSEs and the estimated 95\% ACIs for different choices of $m$ and $k$ for $T=10, \alpha=2$ and $\beta=2$

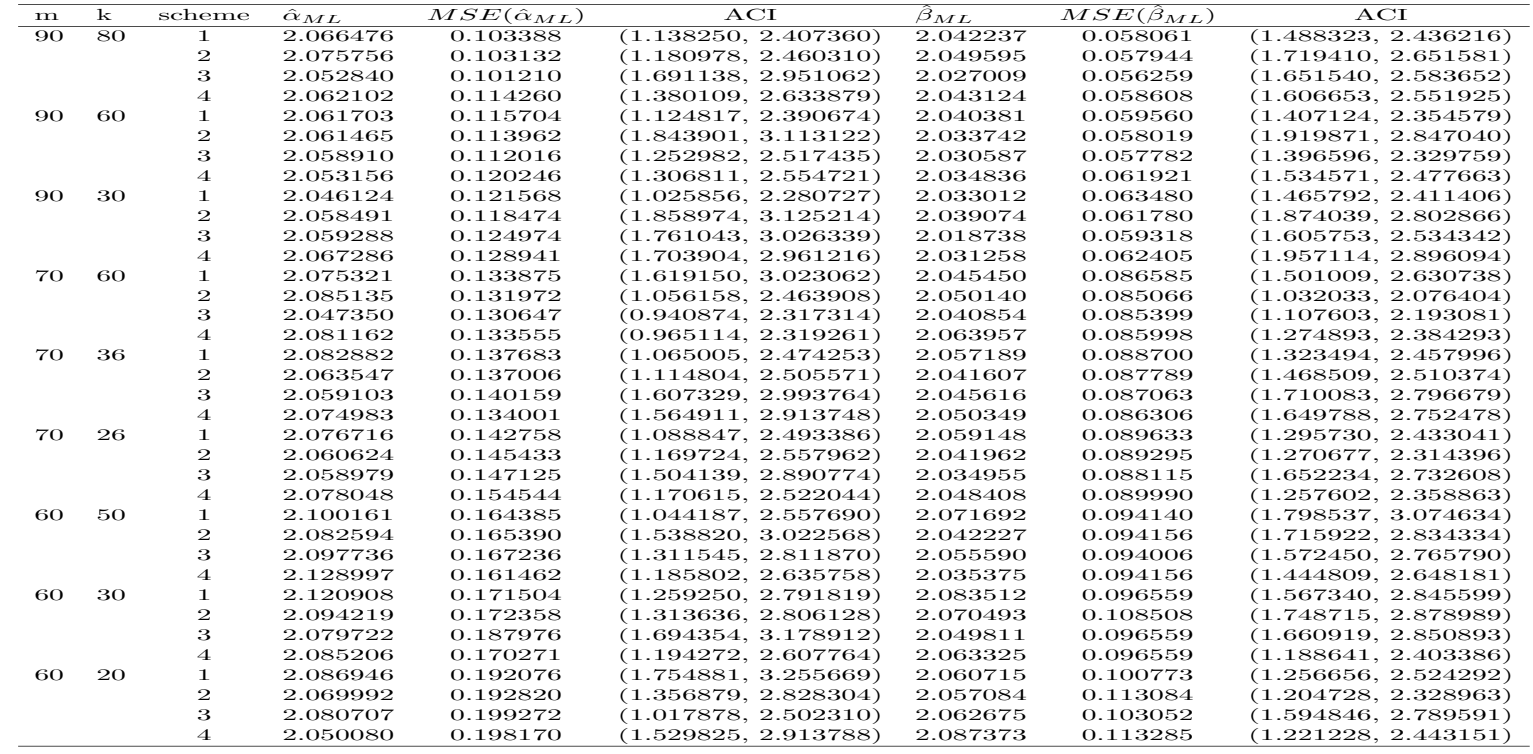

under $S_{m: n}{ }^{(4)}$ are less than that for $S_{m: n}{ }^{(3)}$, i.e., the trend shows a reversal from the small number of removals. The ETNFs decrease with increasing $\beta$, but when a threshold value of $\alpha$ is achieved, ETNFs' plot tends to converge.

The diagrammatic representation for the ETTT and the ETNFs are given in Figure 2 and Figure 3. From Figure 2, the effect of $T$ and $m$ on the ETTT can be seen and it may be noted that as the value of $T$ increases, the ETTT increases; however, the effect of $m$ is very low. Moreover, as $\beta$ increases or $\alpha$ decreases, the ETTT increases. Furthermore, from Figure 3, it can be observed that as $m$ increases, the ETNFs increase, and increasing $T$ increases the ETNFs, but with a slow rate. These magnitude of changes are noted to be decreasing for increasing $\alpha$. However, the ETNFs increase as $\alpha$ increases, but the rate of increase decreases; and when a threshold value of $\alpha$ is achieved, it becomes more or less constant. The value of $\alpha$ is smaller for larger values of $m$ and $T$. Also, as $\beta$ decreases or $\alpha$ increases, the ETNFs increase.

\section{Conclusion}

The estimators of the parameters of EED under the GPH censoring scheme have been obtained. The performances of the estimators have been studied based on their MSEs. From the discussions given above, we may conclude that the MSEs of the proposed estimators are less when the number of removals is small(i.e., $m$ is large), and it occurs at first failure, compared to when removals occur at last failure. Similarly, when removals occur in the mid, the MSEs of the estimators are observed to be less compared to when removals occur at first and last failures. On the other hand, when the number of removals is large(i.e., $m$ is small), the reverse trend is observed. Furthermore, it is observed that the MSEs of both the parameters increase when the minimum number of failures decreases. It is also noted that the ETNFs decrease with an increase in the value of $\beta$ when $\alpha$ is less than 2.5. It is found that the ETTT and the ETNFs can be controlled by parameters, $T$ and $m$. Thus, using the proposed estimator is recommended under the considered censoring scheme with a proper termination time and number of failures. The work can be extended by applying different methods of estimation, such as maximum product spacing, method of least square, etc., to estimate the model parameters. The Bayesian counterpart of the current work may be beneficial. This article would be a great resource for learning about the GPH censoring scheme and its scope. 

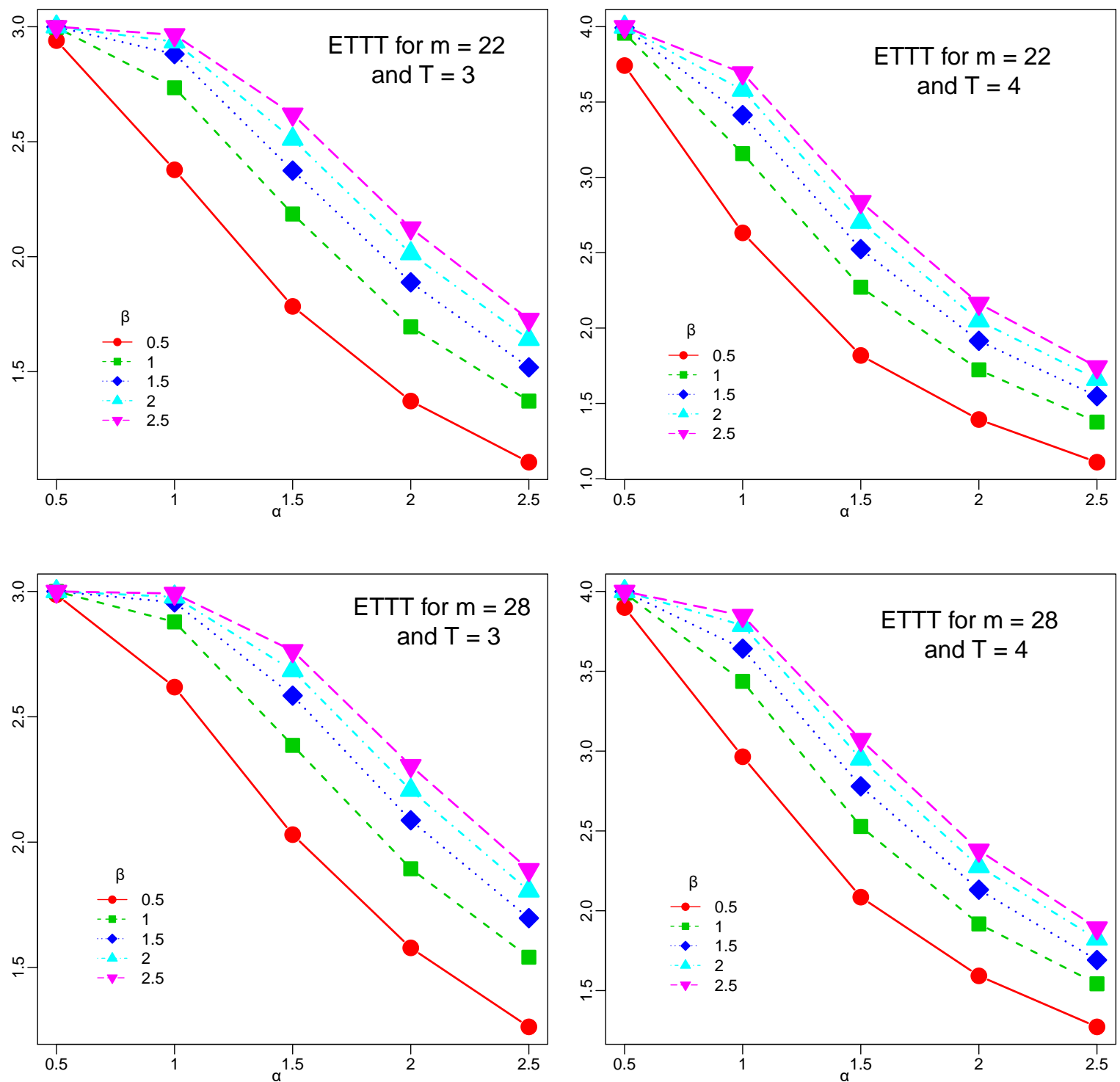

Figure 2: Expected total time on test for different choices of $\alpha, \beta, m$ and $\mathrm{T}$ 

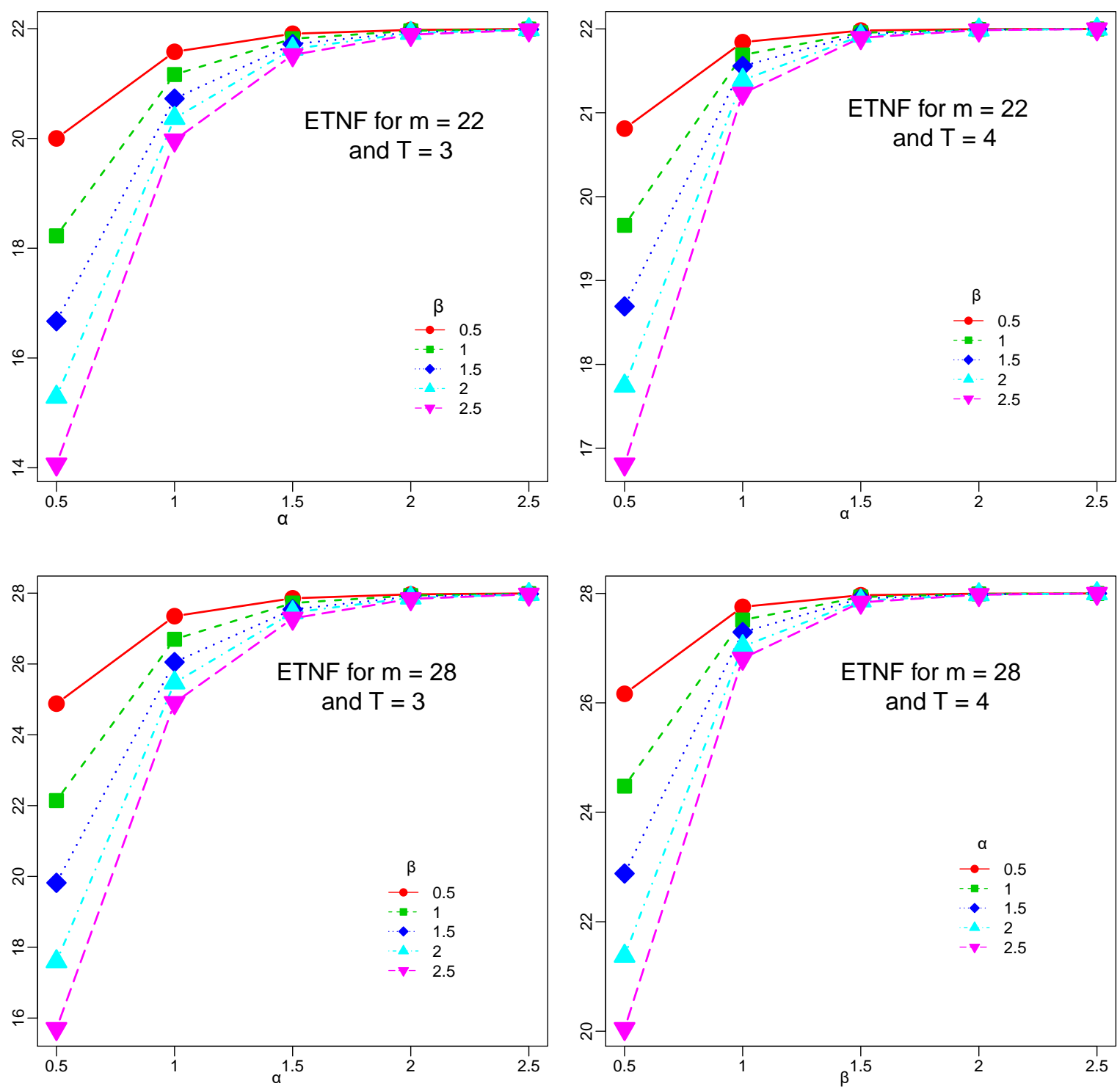

Figure 3: Expected total number of failures for different choices of $\alpha, \beta, \mathrm{m}$ and $\mathrm{T}$ 


\section{Acknowledgment}

We gratefully thank the editor, Dr. Matthias Templ and anonymous referees for their valuable comments on an earlier version that lead to the improved final version.

\section{References}

Awad AM, Alawneh AJ (1987). "Application of Entropy to a Life-Time Model." IMA Journal of Mathematical Control and Information, 4(2), 143-148.

Balakrishnan K (1996). Exponential Distribution: Theory, Methods and Applications. CRC press.

Balakrishnan N (2007). "Progressive Censoring Methodology: An Appraisal." Test, 16(2), 211.

Balakrishnan N, Aggarwala R (2000). Progressive Censoring: Theory, Methods, and Applications. Springer Science \& Business Media.

Childs A, Chandrasekar B, Balakrishnan N (2008). "Exact Likelihood Inference for an Exponential Parameter under Progressive Hybrid Censoring Schemes." In Statistical Models and Methods for Biomedical and Technical Systems, pp. 319-330. Springer Birkhäuser Boston.

Childs A, Chandrasekar B, Balakrishnan N, Kundu D (2003). "Exact Likelihood Inference Based on Type-I and Type-II Hybrid Censored Samples from the Exponential Distribution." Annals of the Institute of Statistical Mathematics, 55(2), 319-330.

Cho Y, Sun H, Lee K (2015a). "Estimating the Entropy of a Weibull Distribution under Generalized Progressive Hybrid Censoring." Entropy, 17(1), 102-122.

Cho Y, Sun H, Lee K (2015b). "Exact Likelihood Inference for an Exponential Parameter under Generalized Progressive Hybrid Censoring Scheme." Statistical Methodology, 23, 18 34 .

Eissa FH, Ahmed HH, Wu SJ (2014). "Statistical Inferences of the Exponentiated Weibull Model under Type II Progressively Censored Data with Random Removals." Wulfenia, 21(3), 335-348.

Epstein B (1954). "Truncated Life Tests in the Exponential Case." The Annals of Mathematical Statistics, 25(3), 555-564.

Ghitany ME, Atieh B, Nadarajah S (2008). "Lindley Distribution and Its Application." Mathematics and Computers in Simulation, 78(4), 493-506.

Gupta RD, Kundu D (1999). "Theory \& Methods: Generalized Exponential Distributions." Australian \& New Zealand Journal of Statistics, 41(2), 173-188.

Gupta RD, Kundu D (2001a). "Exponentiated Exponential Family: An Alternative to Gamma and Weibull Distributions." Biometrical Journal, 43(1), 117-130.

Gupta RD, Kundu D (2001b). "Generalized Exponential Distribution: Different Method of Estimations." Journal of Statistical Computation and Simulation, 69(4), 315-337.

Hemmati F, Khorram E (2013). "Statistical Analysis of the Log-Normal Distribution under Type-II Progressive Hybrid Censoring Schemes." Communications in Statistics-Simulation and Computation, 42(1), 52-75. 
Kaushik A, Maurya SK, Mradula (2016). "Shannon Entropy for Exponentiated Exponential Distributed Data Under Progressively Type-I Interval Censoring Scheme." International Journal of Essential Sciences, 11(1), 37-45.

Kundu D, Joarder A (2006). "Analysis of Type-II Progressively Hybrid Censored Data." Computational Statistics \& Data Analysis, 50(10), 2509-2528.

Shannon CE (1948). "A Mathematical Theory of Communication." Bell System Technical Journal, 27(3), 379-423.

\section{Affiliation:}

Aakriti Pandey

Department of Statistics, Institute of Science

Banaras Hindu University

Varanasi, India-221005

E-mail: akritibhu@gmail.com

Arun Kaushik

Department of Statistics, Institute of Science

Banaras Hindu University

Varanasi, India-221005

E-mail: arundevkauhsik@gmail.com

URL: http://arun-kaushik.github.io/

\section{Austrian Journal of Statistics}

published by the Austrian Society of Statistics

Volume 50

January 2021 http://www.ajs.or.at/

http://www.osg.or.at/

Submitted: 2019-03-14

Accepted: 2020-01-27 